\title{
Deformation Characteristics of Coronary Stents of the Matrix and Continuous Sinusoidal Types in Free Expansion: Computer Simulation
}

DOI: $10.17691 / \mathrm{stm} 2018.10 .2 .03$

Received February 19, 2018

K.V. Ardatov, Engineer';

D.V. Nushtaev, PhD, Engineer ${ }^{2}$

${ }^{1}$ Moscow Aviation Institute, 4 Volokolamskoe shosse, Moscow, 125993, Russia;

${ }^{2}$ Tesis, room 705, 18 Unnatov St., Moscow, 127083, Russia

The aim of the study is to evaluate and compare the stress-strain characteristics of balloon-expandable coronary stents of the matrix and continuous sinusoidal types using the method of imitative mathematical modeling.

Materials and Methods. Based on the reconstructed geometric models, finite element stent models were created and then tested for free expansion using the SIMULIA Abaqus software package (DS SIMULIA, USA).

Results. The ranges of maximum curve and plastic deformations of the stents were identified and the radial recoil values of each of them were determined; in addition, graphs describing the stent diameter as a function of pressure in the balloon were created. When compared the two types of stents had different maximum stress values under the similar external conditions. The continuous sinusoidal stents were found less stressed and having higher margins of strength on expansion as compared with the matrix stents.

Key words: balloon-expandable coronary stents; mechanical characteristics of stents; finite element analysis; SIMULIA Abaqus; matrix type stents; continuous sinusoidal stents.

\section{Introduction}

Balloon-expandable coronary stents are widely used for minimally invasive (endovascular) treatment of coronary heart disease. At the current moment, there is a large variety of coronary stents on the market; among them, the most common are matrix type stents that are manufactured by laser machining of an all-metal tube (matrix) and continuous sinusoidal type stents that are manufactured by winding a sinusoidal wire around a cylindrical core.

One of the main requirements is to ensure mechanical strength and integrity of the stent structure at all stages of its deployment, including the stage of additional expansion needed to maintain the lumen diameter sufficient for adequate blood flow [1]. In this regard, a comparative assessment of the mechanical characteristics of stents is important for both developers and manufacturers because it lays the ground for further design optimization; it is also useful for cardiologists - as it helps to better evaluate the functional characteristics of a stent and choose the stent most suitable to a specific clinical situation.

One of the main tools for analyzing and predicting the behavior of various stents in different clinical situations is the imitative simulation based on the finite element analysis [2-4]. This approach allows one to significantly reduce the time and cost of developing new stents, as well as to improve the functional characteristics of existing stents by using multi-objective design optimization $[5,6]$.

The aim of the study was to compare the stressstrain characteristics of balloon-expandable coronary stents of the matrix type and the continuous sinusoidal type by using the method of imitative mathematical modeling.

\section{Materials and Methods}

Coronary stents. The deformation characteristics of three metallic balloon-expandable stents available in the Russian market were studied: a sinusoidal type stent ST Brig (Stentex, Russia) and two matrix type stents: Sinus (Angioline, Russia) and NexGen (Meril Life, India).

The schematic view of the cells and connectors in the stent structures is shown in Figure 1; information on the major sizes and the alloy brands is given in Table 1 .

Based on the optical and scanning electron microscopy measurements, geometric models of the repeating cellular elements were created and the number of these elements in the axial and radial directions was determined. These cells were then used to draw planar sketch that reflected the crimped configuration of each stent. The solid parametrized geometric models were

Corresponding author: Dmitry V. Nushtaev, e-mail: nyshtaev.vfb@rambler.ru 


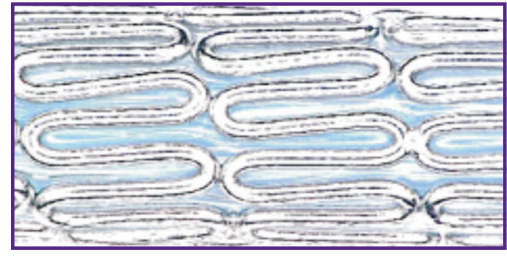

ST Brig

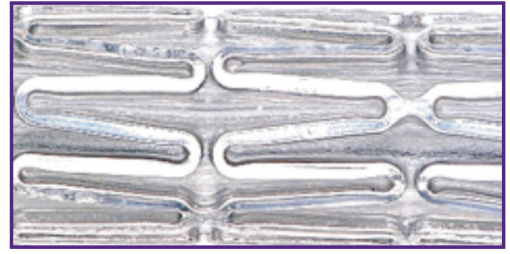

Sinus

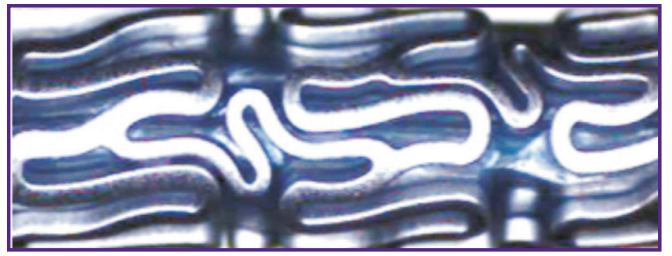

NexGen

Figure 1. General view of cells and connectors in the examined coronary stents

Table 1

General characteristics of stents used in this study

\begin{tabular}{lccccc}
\hline \multicolumn{1}{c}{$\begin{array}{c}\text { Brand } \\
\text { name }\end{array}$} & Manufacturer & Type & Alloy brand & $\begin{array}{c}\text { Rated dimensions/ } \\
\text { pressure }\end{array}$ & Section geometry/size \\
\hline ST Brig & Stentex, Russia & Continuous sinusoidal & Co-Cr MP35N & $2.5 \times 22 \mathrm{~mm} / 9 \mathrm{~atm}$ & Circle $/ 90 \mu \mathrm{m}$ \\
\hline Sinus & Angioline, Russia & Matrix & Co-Cr L605 & $2.5 \times 18 \mathrm{~mm} / 9 \mathrm{~atm}$ & Rectangle $/ 50 \times 80 \mu \mathrm{m}$ \\
\hline NexGen & Meril Life, India & Matrix & Co-Cr L605 & $2.5 \times 13 \mathrm{~mm} / 9 \mathrm{~atm}$ & Rectangle $/ 65 \times 90 \mu \mathrm{m}$ \\
\hline
\end{tabular}

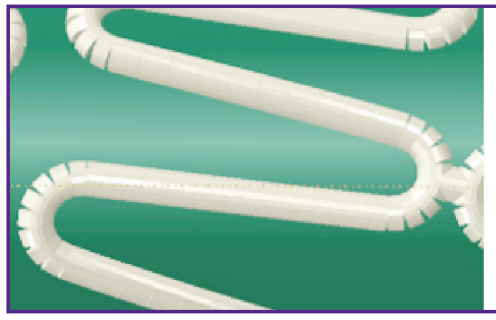

ST Brig

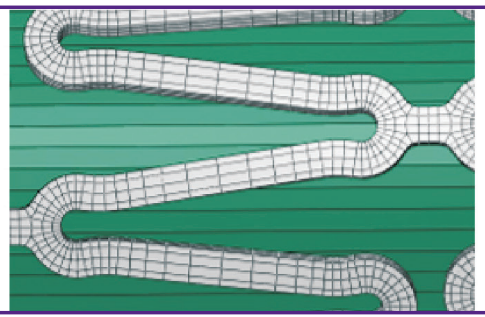

Sinus

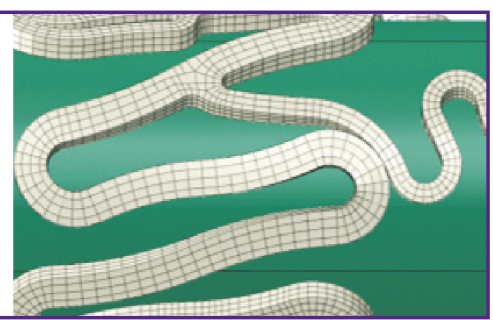

NexGen

Figure 2. Fragments of the finite element stent models

obtained by wrapping the created planar mesh into cylindrical shape of relevant diameter.

Numerical simulation. Simulation of free expansion of coronary stents was performed using the SIMULIA Abaqus software package based on the finite element analysis. We used the solver with the implicit integration of the motion equations with time (Abaqus/Standard). The solution was obtained in a geometrically and physically nonlinear setting with the presence of multiple contact interactions between the individual stent cells and the surface of the inflation balloon.

Each calculation model included a model of the stent and a model of the inflation balloon. The creation of finite element models of the stents was carried out on the basis of the reconstructed geometric models.

For the matrix stent (Sinus and NexGen) modeling, three-dimensional linear brick element with one integration point (C3D8R) were used [7].

Finite element models of ST Brig stents having a circular sectional profile created with three-dimensional elements are inefficient from a computational point of view and are limited by the resources of the available hardware. Considering that, the planar sketch was approximated by the beam end elements (B31) according to the Timoshenko's version [7]. The beam elements were characterized by a circular profile with a cross-section of radius $r=45 \mu \mathrm{m}$.

Each stent model has its own equivalent size of finite elements. We utilized the technique of local compaction of a computational mesh in the points of expected stress concentrators namely the cell peak and the points of attachment of the longitudinal connectors. The final configuration of the finite element models was determined taking into account the relationship between the size/shape of the finite elements and the simulation result. Fragments of the created computational models are shown in Figure 2.

In each of the models, the "inflation balloons" are represented by cylindrical shells with initial diameters identical to the internal diameters of the respective stents. To simplify the physical model and minimize the computation costs, we skipped the modeling of folded configurations of the balloons. In the case of the stressstrain evaluation of stents with free expansion (without the vessel), this simplification is acceptable because it has little effect on the simulation result $[5,8]$. Finite element shell models were developed on the basis of superficial, absolutely compliant SFM3D4 elements [7], 
which provided a correct assessment of the integral stiffness of the stent.

The stents in question are made of highstrength and high-plastic cobalt-chromium alloys. We described the nonlinear behavior of these materials by applying an elastic-plastic model with isotropic hardening. The Mises' fluidity surface and the associated law of plastic flow were used. The mechanical constants of the selected model were chosen based on the alloy deformation diagrams obtained in the static uniaxial stress-to-destroy experiment at room temperature [9, 10] (Table 2).

At the initial stage of the simulation, the cylindrical shell of the inflation balloon was located inside the non-attached stent (Figure 3). To simulate stress transmission, we used a coefficient of friction of 0.1 to describe the contact interaction between the outer surface of the inflation balloon and the inner surface of the stent. To ensure the implementation of contact restrictions, the penalty method was applied. The default: "penalty" (contact) stiffness is based on the stiffness of the elements in the contact area. As a result, the mutual penetration value is lower than the elastic deformations of the initial element in a direction perpendicular to the contact surface.

The expansion was simulated by determining the radial displacement along the inflation balloon $U_{R}$ : $0.63 \mathrm{~mm}$ for ST Brig, $0.59 \mathrm{~mm}$ for Sinus, and $0.69 \mathrm{~mm}$ for NexGen. The adjusted value provides the stents expansion to their rated dimensions (see Table 1). After completion of this process, the inflation balloon assumes the initial position, which allows for elastic unloading of the stent. Elastic unloading is accompanied by a reduction of the current diameter, the so-called radial recoil $\left(D_{\text {rec }}\right)$. Radial recoil is an important functional characteristic of the stent, since it directly affects the degree of its adherence to the vascular wall [11]. Its value was assessed from the data on the stent diameter under the maximum load $\left(D_{\text {load }}\right)$ and the diameter after unload $\left(D_{\text {unload }}\right)$ :

$$
D_{\text {rec }}=\frac{D_{\text {load }}-D_{\text {unload }}}{D_{\text {load }}} 100 \%
$$

Based on the simulation results, curves of the current diameter of the stent versus the pressure in the balloon were plotted. The values of the current diameter were calculated through the radial movements of the crosspoints of the cylindrical shell of the inflation balloon. The values of the internal pressure $(p)$ were obtained from mathematical processing of the support reaction forces arising in the balloon during its opening:

$$
p=S^{-1} \sum_{i=1}^{n} f_{i}
$$

where $f$ - cross-point reaction forces in the balloon; $S$ the inner surface area of the stent; $n-$ the consecutive numbers of the balloon cross-points that are in contact with the stent.

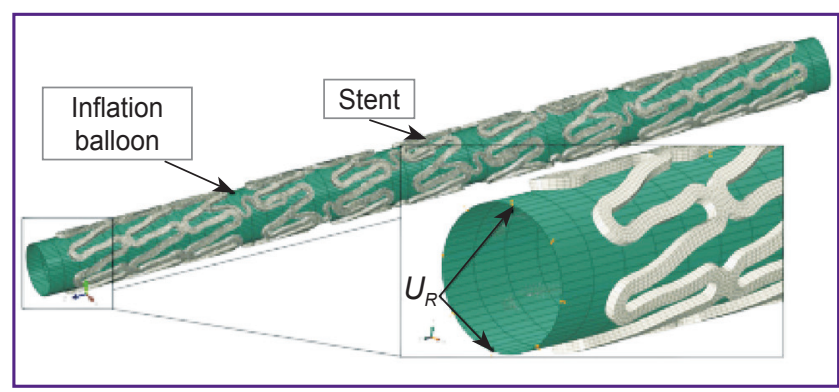

Figure 3. General scheme of the computational model (exemplified by the NexGen stent)

\section{Results and Discussion}

As a result of the present free expansion simulation, the deployed stents models were obtained and their deformation characteristics were analyzed: those were the distribution of mechanical stresses and equivalent plastic deformations for the ST Brig (Figure 4), Sinus (Figure 5), and NexGen (Figure 6) stents. The Figures show that the cell peaks and the cross-points of the longitudinal connectors are the most heavily stressed elements. Based on the pattern of the major stresses, the stress-strain state of these points (with a sufficient degree of approximation) can be compared with the pure bending of the curved beam by concentrated moments. The most critical for the integrity of the whole structure are the zones of maximum stresses at the inner radii of the cell apex rounding, where the greatest positive (tensile) stresses develop; those can even lead to cracking if material defects are present.

Figure 4 shows the distributions of the tensile (a) and compressive (b) stresses to the state of recoil at the extreme points of integration located near the ST Brig stent surface. For the outer side (relative to the bending) of the cells, compressive stresses of $\sigma_{\text {max }}^{-}=633 \mathrm{MPa}$ are characteristic, and for the inner side, tensile stresses of $\sigma_{\max }^{+}=638 \mathrm{MPa}$ are typical as well. It should be noted that the mechanical stresses in this stent do not exceed the strength of the material at all points of the frame $\left(\sigma_{y}=930 \mathrm{MPa}\right)$, and also provide a strength margin of about $30 \%$.

The distribution of the major tensile and compressive stresses in the Sinus and NexGen stents is shown in Figures 5 and 6 . As in the previous case, for the outer side (relative to the bending) of the cells, the compressive stresses are characteristic: $\sigma_{\max }^{-}=-910 \mathrm{MPa}$ 


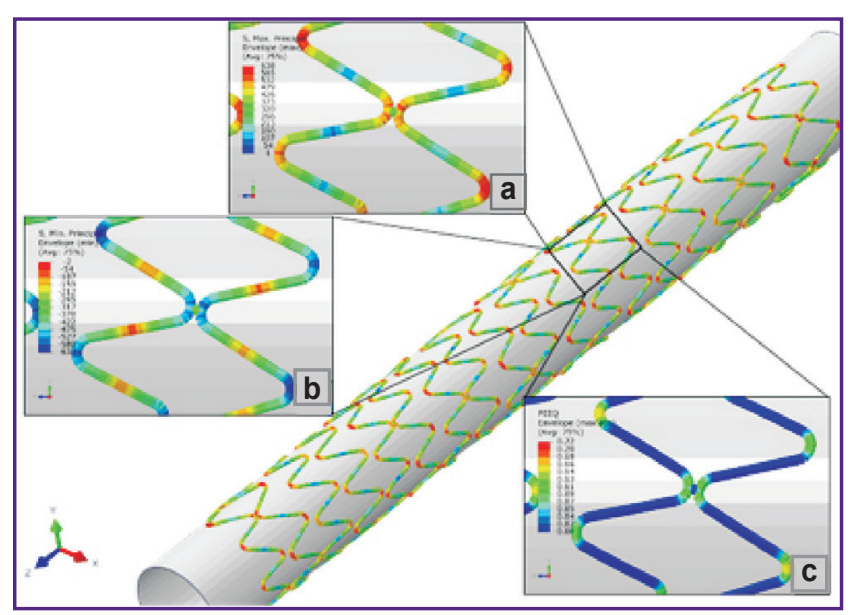

Figure 4. Stress-strain state of the ST Brig stent:

(a) distribution of tensile stresses (MPa); (b) distribution of compressive stresses (MPa); (c) distribution of equivalent plastic deformations

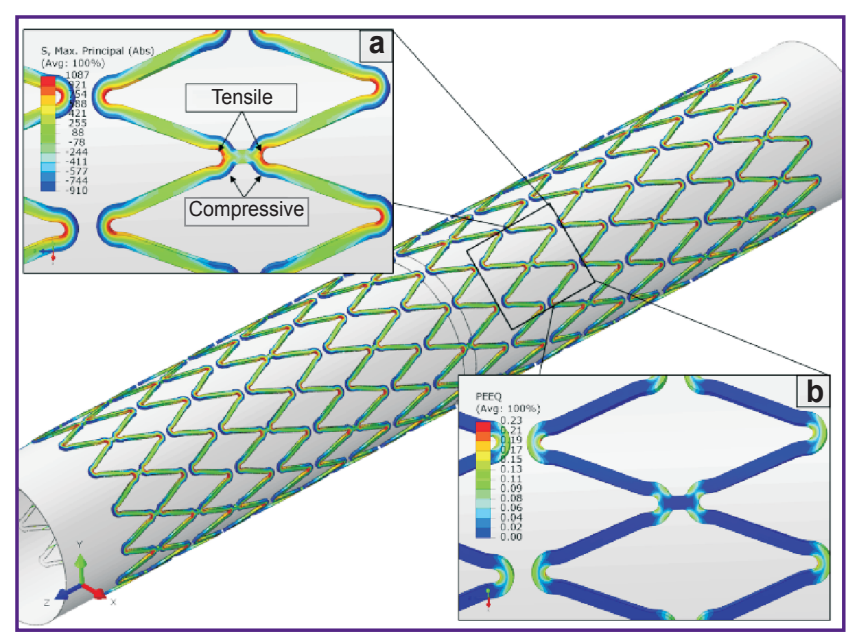

Figure 5. Stress-strain state of the Sinus stent:

(a) distribution of tensile and compressive stresses (MPa);

(b) distribution of equivalent plastic deformations

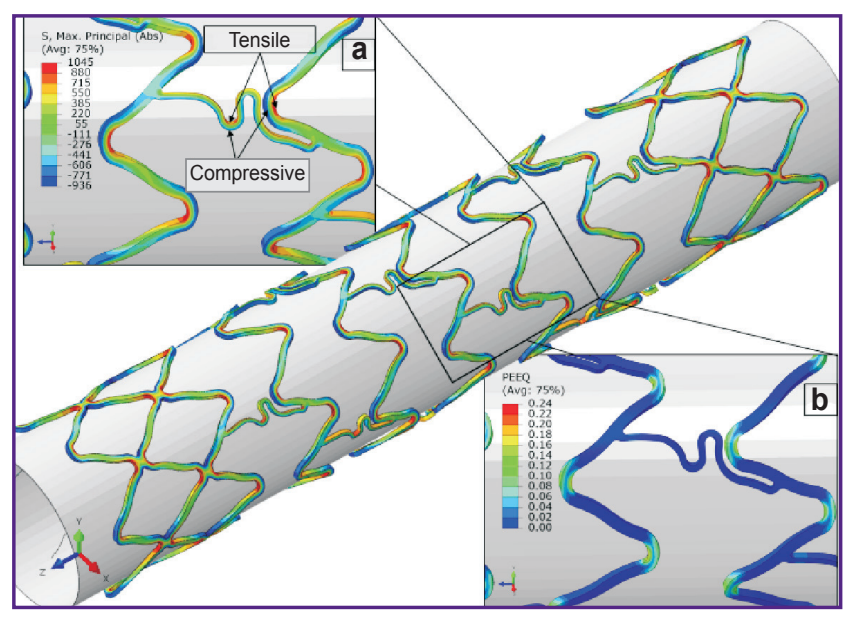

Figure 6. Stress-strain state of the NexGen stent:

(a) distribution of tensile and compressive stresses (MPa);

(b) distribution of equivalent plastic deformations for Sinus and $\sigma_{\max }^{-}=-939 \mathrm{MPa}$ for NexGe. For the internal side, the tensile stresses are $\sigma_{\text {max }}^{+}=1087 \mathrm{MPa}$ for Sinus and $\sigma_{\max }^{+}=1045 \mathrm{MPa}$ for NexGen. The level of maximum stresses in these stents is close to the strength limit of the material $\left(\sigma_{y}=1097 \mathrm{MPa}\right)$, which indicates the closeto-limit work of the material at the cell peaks and the limited possibilities for further expansion of these stents.

Irreversible expansion of balloon-expandable stents is the result of plastic deformation of the stent cells in areas adjacent to their peaks where plastic hinges are formed. The straight sections of the cells serve levers for the transmission of circumferential forces in the cylindrical structure of the stent to the plastic hinges in the areas adjacent to the peaks. Thus, the size and distribution of the plastic deformations accumulated during the expansion phase determine the ability to retain the initial shape of the stent. The maximal equivalent plastic deformations are localized at the cell peaks and reach $21.5 \%$ for ST Brig, $23.2 \%$ for Sinus, and $24.4 \%$ for NexGen.

Unloading the structure after depressurizing the balloon is accompanied by a drop in the stress magnitude and a decrease in the stent diameter. At that stage, the radial recoil values were $4.4 \%$ for ST Brig, $5 \%$ for Sinus, and $3.2 \%$ for NexGen, which is in agreement with the manufacturers' data.

Curves depicting changes in the stent diameter upon changes in the static pressure inside the inflation balloon are shown in Figure 7. The curves consist of two distinct, almost linear, sections. At the starting section $(0<p<4.2$ atm for ST Brig; $0<p<4.8$ atm for Sinus; $0<p<7.5 \mathrm{~atm}$ for NexGen), the stents have a relatively high radial stiffness, which is due to the work of the material in the cell apical areas with high rigidity values. Upon reaching the target expansion pressure (4.2 atm for ST Brig; 4.8 atm for Sinus; 7.5 atm for NexGen) the radial stiffness of the stents drops significantly due to the switch of the cell apical materials to work under the elastic-plastic conditions and the formation of elasticplastic hinges. The pressure required to open the stents up to their rated dimensions without considering the balloon elasticity was $5.5 \mathrm{~atm}$ for ST Brig, $5.9 \mathrm{~atm}$ for Sinus, and $11.0 \mathrm{~atm}$ for NexGen.

Thus, the comparative evaluation and analysis of the stress-strain states of the ST Brig, Sinus, and NexGen coronary stents with free expansion demonstrated that the tested stents had different levels of maximum stresses under similar external conditions.

In all cases, the most heavily stressed elements were the peaks of the cells and the points where the longitudinal connectors were attached; the maximum stresses on the inner radii of the apical rounding, where the greatest tensile stresses develop, are the most critical for the integrity of the entire structure. At the same time, the operating stresses at the peaks of the Sinus and NexGen stent cells are close to the steel strength limits, which should be taken into account during the post-dilatation procedure (the extra 
Figure 7. Dependence of the current stent diameter on the intra-balloon pressure

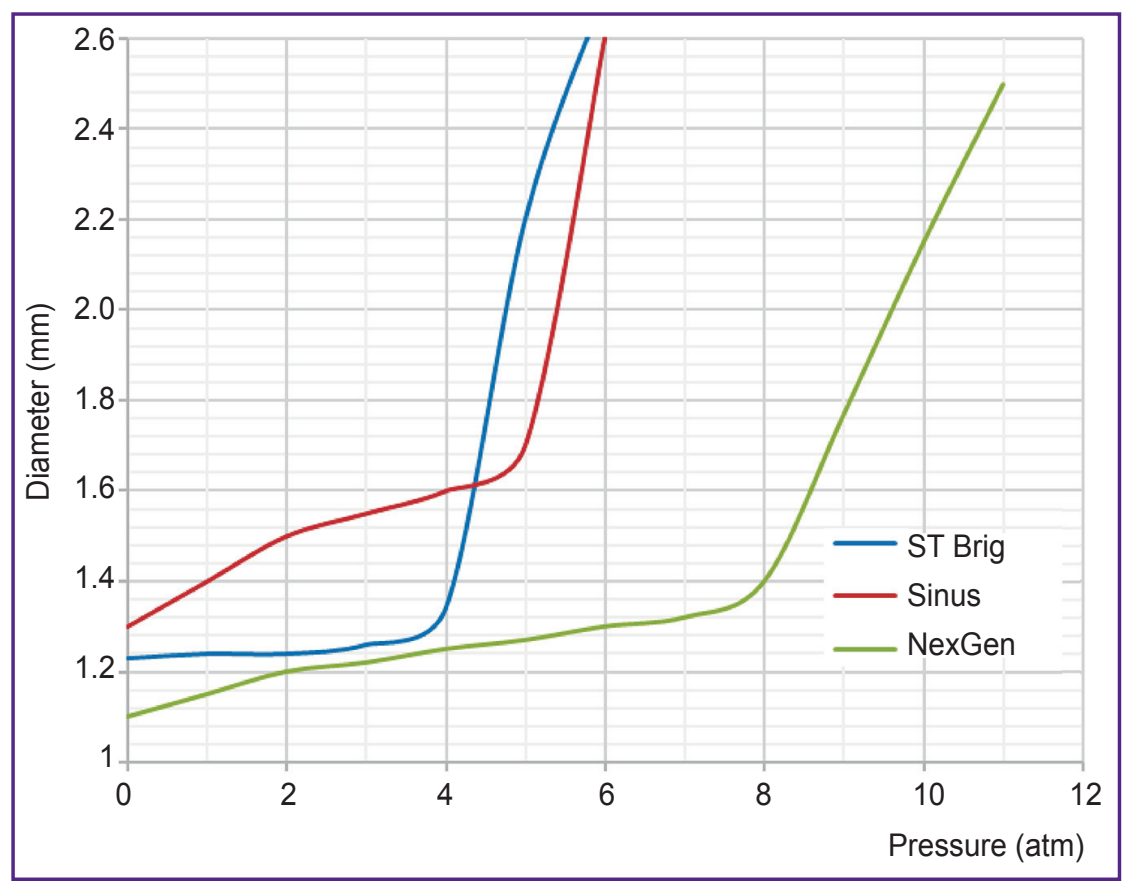

expansion of the stent to achieve better fit of its beams to the vessel wall).

The continuous sinusoidal design of the ST Brig stent is the least stressed system with a minimum stress level; this structure provides the maximum strength margin as compared with two other models tested in this study.

\section{Conclusion}

The proposed analysis of the mechanical strength of balloon-expandable stents using mathematical modeling makes it possible to compare the models of coronary stents made by different technologies. The obtained data can be used in the development of new types of stents and can help assess the possibility of using a particular model in a specific clinical case.

Research funding and conflict of interest. The study was not funded by any sources, and there are no conflicts of interest related to this study.

\section{References}

1. FDA Center for Devices and Radiological Health. Non-clinical engineering tests and recommended labeling for intravascular stents and associated delivery systems. Guidance for Industry and FDA Staff. 2010.

2. Nushtaev D.V. Use of SIMULIA Abaqus software for solving biomechanics problems. SAPR i grafika 2014; 9(215): 114-117.

3. Nushtaev D.V., Zhavoronok S.I., Klyshnikov K.Y.,
Ovcharenko E.A. Combined numerical and experimental investigation of the deformed state and buckling of the meshed cylindrical shell subjected to the axial compression. Trudy MAI 2015; 82: 9.

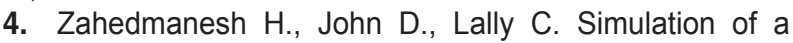
balloon expandable stent in a realistic coronary artery determination of the optimum modelling strategy. $J$ Biomech 2010; 43(11): 2126-2132, https://doi.org/10.1016/j.jbiomech. 2010.03.050.

5. Shankaran K., Karrupaswamy S. Parameterization and optimization of balloon expandable stent. SIMULIA Community Conference 2012; p. 1-10.

6. Wang W.Q., Liang D.K., Yang D.Z., Qi M. Analysis of the transient expansion behavior and design optimization of coronary stents. J Biomech 2006; 1(39): 21-32, https://doi. org/10.1016/j.jbiomech.2004.11.003.

7. Abaqus Verification Guide (6.14). Dassault Systemes Simulia USA; 2015.

8. Gervaso F., Capelli C., Petrini L., Lattanzio S., Di Virgilio L., Migliavacca F. On the effects of different strategies in modelling balloon-expandable stenting by means of finite element method. J Biomech 2008, 41(6). 1206-1212, https://doi.org/10.1016/j.jbiomech.2008.01.027.

9. Poncin P., Proft J. Stent tubing. understanding the desired attributes. Materials \& Processes for Medical Devices Conference; 2003.

10. Poncin P., Millet C., Chevy J., Proft J, comparing and optimizing $\mathrm{Co}-\mathrm{Cr}$ tubing for stent applications. Materials \& Processes for medical Devices; 2004.

11. Migliavacca F., Petrini L., Montanari V., Quagliana I., Auricchio F., Dubini G. A predictive study of the mechanical behaviour of coronary stents by computer modelling. Med Eng Phys 2005; 27(1): 13-18, https://doi.org/10.1016/j. medengphy.2004.08.012.

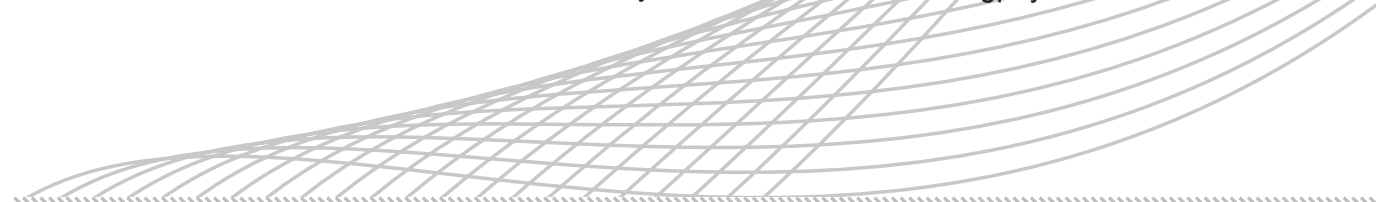

